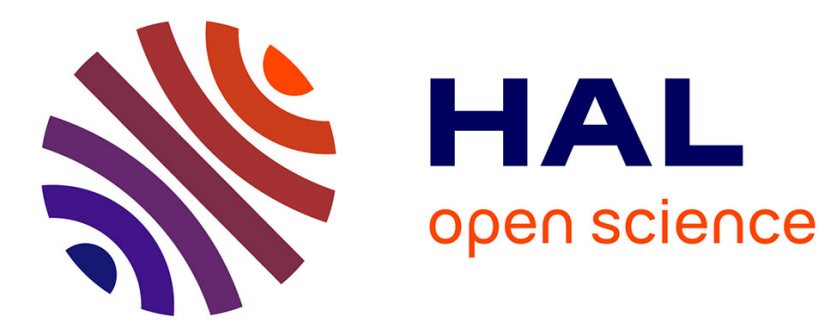

\title{
On physicalism and downward causation in developmental and cancer biology
}

Ana M. Soto, Sonnenschein Carlos, Paul-Antoine Miquel

\section{To cite this version:}

Ana M. Soto, Sonnenschein Carlos, Paul-Antoine Miquel. On physicalism and downward causation in developmental and cancer biology. Acta Biotheoretica, 2008, 56 (4), pp.257-274. hal-00714937

\section{HAL Id: hal-00714937 \\ https://hal-univ-tlse2.archives-ouvertes.fr/hal-00714937}

Submitted on 30 Aug 2013

HAL is a multi-disciplinary open access archive for the deposit and dissemination of scientific research documents, whether they are published or not. The documents may come from teaching and research institutions in France or abroad, or from public or private research centers.
L'archive ouverte pluridisciplinaire HAL, est destinée au dépôt et à la diffusion de documents scientifiques de niveau recherche, publiés ou non, émanant des établissements d'enseignement et de recherche français ou étrangers, des laboratoires publics ou privés. 


\title{
On physicalism and downward causation in developmental and cancer biology
}

\author{
A. M. Soto*\# \\ C. Sonnenschein*\# \\ P.A. Miquel $\#+$
}

*Tufts University School of Medicine, Department of Anatomy and Cellular Biology. Boston, MA 02111, USA

+Centre de Recherche et d'Histoire des Idées, Université de Nice, France

\#Centre Cavaillès, Ecole Normale Supérieure, Paris, France

Corresponding author: P.A. Miquel

Paul-Antoine.MIQUEL@unice.fr

\begin{abstract}
:
The dominant position in Philosophy of Science contends that downward causation is an illusion. Instead, we argue that downward causation doesn't introduce vicious circles either in physics or in biology. We also question the metaphysical claim that "physical facts fix all the facts." Downward causation does not imply any contradiction if we reject the assumption of the completeness and the causal closure of the physical world that this assertion contains. We provide an argument for rejecting this assumption. Furthermore, this allows us to reconsider the concept of diachronic emergence.
\end{abstract}




\section{Introduction}

Physicalism and antireductionism in biochemistry and in molecular biology are topics of renewed current interest. Rosenberg and Kaplan attempted to reconcile them (Philosophy of Science (2005). For this purpose they developed an argument whereby they reject the downward causation by undermining one of the epistemological pillars of biology: i.e., the very distinction in Mayr's view (1961), between the question "how?" that has a meaning in biochemistry as well as in the physical sciences, and the question "how come and why" that has sense only in evolutionary biology. This duality introduces a gap between physics and biology. Rosenberg and Kaplan attempt to close this gap by trying to force a biological concept, that of natural selection, into the realm of physics. To do so, they claim that the principle of natural selection is an underived law about physical systems; in other words, it is already present in the field of physics and chemistry. According to the authors, the difference between physical and biological objects is not due to fitness, a notion similar to entropy, but rather due to reproduction.

In order to be swayed to Rosenberg and Kaplan's position, one should acknowledge that fitness is a concept that belongs to physics. And yet, in their view, natural selection is also a supervenient property like entropy in statistical mechanics. However, this doesn't involve any ontological implication, because it doesn't give rise to irreducible levels of properties and entities; it just reveals the limits of our knowledge instruments. Thus, even if they believe that biological theories are not reducible to physical ones in an epistemic sense, this assertion doesn't lead to any "implicit or explicit commitment to metaphysical or ontological differences" between physics and biology. Like Kim (1993), they defend a "vindicating physicalism" based on the physical determination thesis ("physical facts fix all the facts") and on the ontological principle of completeness and of causal closure of the physical world. Consequently, they reject downward causation because it would not be compatible with physicalism's commitment (2005).

We will argue to the contrary that downward causation does not introduce vicious circles either in physics or in biology. Downward causation does not imply any contradiction once we reject Rosenberg and Kaplan's assumption concerning the completeness and the causal closure of the physical world. Such an ontological claim introduces the God's Eye fallacy in ones' metaphysical vision of Nature (Putnam, 1990). By rejecting this claim we are allowed to reconsider the concept of diachronic emergence.

\section{1- Fitness as a physical property}

Rosenberg and Kaplan claim that the argument of "substrate-neutrality" of fitness doesn't prove that the principle of natural selection (PNS) is an "underived" law of biology. According to their view, the law of entropy in macroscopic thermodynamics is also substrate neutral, and yet it can be reduced to the growth of the logarithm of the complexion number up to the state of equilibrium in a closed system where all accessible microstates are equiprobable. Therefore, they ponder whether a similar reduction could be made for fitness. 
Let us explore the argument of substrate neutrality (Dennett, 1996: 50-51). In Dennett's view, substrate neutrality is linked to the idea of an algorithm as a mechanical procedure, an idea coming from Turing, Gödel and Von Neumann. We acknowledge the significance of this notion in the construction of classical molecular biology that derives in turn from the concept of microcode in Schrödinger's view. However, Dennett uses it in the context of evolutionary biology. If he is right, natural selection acts as a mindless and mechanical meta-engine that modifies biological engines, which in turn generate the growth of complexity and adaptability. Yet, adaptability is not an equilibrium state that can be predicted. It is, instead, the result of an instantiation of a mindless algorithm under rules of blind design, which makes sense only retrospectively because it is a product of evolution. Dennett proposed this concept of "accumulation of design" in contrast to the vision of a creative order that produces novelty from nothing that he attributes to Gould. In Dennett's view, natural selection would be a "crane" and not a "skyhook".

According to Dennett, natural selection adds logical constraints to the physical ones. Thus, a distinction must be made between the physical structure in which the algorithm is implemented on the one hand, and the function that it fulfils, on the other. And here is where Dennett introduces the principle of the substrate neutrality (PSN), founded on the idea that "the power of the procedure is due to its logical structure" which can be realized in very different substrates (Dennett, 1996).

Rosenberg and Kaplan are cautious about this definition of the PSN. According to their views, there cannot be any real "biological kinds", any real difference of level between physics and biology, even if one accepts that "biology relies on a theory that is not reducible to a more fundamental physical theory" (Rosenberg and Kaplan , 2005). This could be the case, only if all natural facts (chemical, biological) are fixed as physical ones. Given that this is true, the distinction between physics, biology and psychology is only semantic due to human "conceptual incommensurabilities or epistemic limitations," and would have no ontological implications. This point is also emphasized by $\operatorname{Kim}\left(1999^{2}\right)$.

According to Rosenberg and Kaplan, entropy seems to be also a "supervenient" property, and yet it can be reduced to statistical disorder" ${ }^{3}$. The fact that temperature is not varying at the equilibrium state is founded on the ergodicity hypothesis ${ }^{4}$ : thus, the system will continually (eternally?) instantiate all these states in compatibility with its temperature. In this case, one must presume, first, that such a system is closed; second, that a gas is composed "of concrete constituents with mass and velocity"; and third, that such a hypothesis is true "in the limit of infinite time (2005)". Rosenberg and Kaplan know that this sentence has no physical sense. But for them, idealisations are just showing the semantic imperfections of theories as instruments. They have no ontological meaning. Mechanical facts fix thermodynamic facts.

\footnotetext{
1 “The skyhook concept is perhaps a descendant of the deus ex machina of ancient Greek dramaturgy: when second-rate playwrights found their plots leading their heroes into inescapable difficulties they were often tempted to crank down a god into the scene, like Superman, to save the situation supernaturally" (Dennett, 1996, 74).

2 "That is, we interpret the hierarchical levels as levels of concepts and descriptions, or levels within our representational apparatus, rather than levels of properties and phenomena in the world".

${ }^{3}$ Normally, "substrate neutrality" and "supervenience" are not compatible with a strong reductionism, since supervenient properties cannot be deduced form physical ones: "no actual derivation may be effected, owing to the famous "many-one" relation between the physical supervenience base and the biological properties to which the PNS adverts" (Rosenberg and Kaplan, 2005).

${ }^{4}$ A system of molecules will assume in a long period of time all conceivable microstates that are compatible with the conservation of energy.
} 
Why cannot this also be the case with natural selection? Of course, this PNS needs replicative entities that are present in a non-replicative realm. It becomes difficult to understand how PNS can have a meaning before the existence of replicative entities. Yet the authors pursue the idea, already used by Cairn Smith (1982), to explain the transition between the prebiotic and the biotic world. Under certain chemical conditions, we can observe that more stable molecules can be produced which have a greater chance to be preserved and to lead to a more stable macrostructure. One could imagine connections between such structures and the origin of replication, and that a specific equilibrium could be reached between unstable replicative entities and stable non-replicative molecules. Accordingly, the PNS could be applied to stable and unstable molecules in an abiotic world, as it happens with replicative biological entities. Thus, for Rosenberg and Kaplan, chemical facts would fix biological facts.

Rosenberg and Kaplan also conclude that downward causation is incompatible with their physicalist credo. On this point, their view regarding the causal agency of supracellular structures differs from that of P. Kitcher. In Kitcher's view (1984) the formation of a gap among cells would affect the density gradient of a molecular gene product during wing morphogenesis that would be responsible for the formation or malformation of the wing. According to Rosenberg and Kaplan, the supracellular geometrical structure is a supervenient property affecting the genes, and fixed by the evolution of a chemical milieu controlled by the PNS. Thus, the downward causation effect would be a product of this "wholly physical process."

Their view assumes that natural selection is a "physical law" on the same footing as that of entropy: " $(x)(y)(E)$ [If $x$ is fitter than $y$ in environment $E$ at generation $n$, then probably there is some future generation $n$ ' after which $x$ has more descendants than $y$ ] (Rosenberg Kaplan, 2005)." We disagree with this view.

To begin with, can PNS be expressed as an algebraic formula of the kind the authors propose? If we follow their reasoning, one may assume that the change in a genetic pool between a generation $n$ and a future generation $n$ ' is predictable by a formal analysis. This was precisely Fisher's credo in Genetical Theory of Natural Selection (Fisher, 1930). Fisher accepted the assumption of gene additivity, and thought that the value of fitness depended solely on a change in a gene frequency. However, in 1932, Sewall Wright claimed that such an assumption was unrealistic, since genes and alleles are interacting by epistasis, pleiotropy and dominance (S.Wright, 1932). He insisted on the division of a genetic population in demes far away from panmictic equilibrium, and on the role of the sampling effect in each deme. He concluded that in a "shifting balanced theory of evolution", the change in fitness value could be illustrated by adaptive landscapes. In this case, fitness was not predictable from a formal analysis of gene frequency. For Wright (1932, 1977, 1980), in evolutionary biology, what comes first is creativity and novelty, not prediction. Moreover, scientific explanations in population genetics are not fully quantitative.

Second, if the PNS would be a basic law of physics or of chemistry, it should be operational at the lower-level systems of molecules, but this does not imply automatically, as Rosenberg and Kaplan assume, that it is also operational "at each level of organisation of matter" (2005).

Third, seventy years after Wright's observation, the unpredictability problem remains unsolved, concerning the applicability of the PNS. For instance, in Kauffman's simulations on Boolean networks, even when initial conditions and logical laws are chosen randomly, the 
reproduction of the same computations with the same initial conditions produce the same results. Then, it is possible to give an "epistatic" value $\mathrm{K}$ to the fitness contribution of a given gene by other genes. His NK model is an idealisation of an organism constituted by $\mathrm{N}$ genes. When a specific value is assigned to $\mathrm{K}$, the evolution of a multipeaked fitness landscape can be simulated. However, the properties that emerge from the use of such computations are generic features, in other words, they are not described by the initial conditions. In order to predict an outcome, one must first reproduce a situation, and not the contrary. That is probably why Kauffman uses the concept of emergence. Natural selection cannot be understood as a law by which a prediction can directly be made. Natural selection acts from the outside, like "an invisible hand" (Kauffman, 2000, 204) on a system which is not completely defined by its internal constraints (such as the value of the connectivity parameter $\mathrm{K}$, the number $\mathrm{N}$ of Boolean automata symbolising the genes, the iteration mode, etc). From this perspective, the PNS would be a "contextual open property". It could not be characterised as a fundamental law of physics, because it is neither a property of the elements composing a system (the automata, for instance) or the relations between these elements, nor a property of a system determined as a whole (Bunge, 2003, 13). Such Boolean networks have specific generic properties that could occur without assuming that external forces are acting to give each microelement a fitness value. On the contrary, in this model, such a system is a part of a much more complex system that is not self-similar. Kauffman developed this explanation of the properties of a system by integrating it into a more complex one. He tried to simulate coevolution (Kauffman, 2000).

Finally, Rosenberg and Kaplan conclude, that PNS is "a basic law of chemistry", and not of physics, because "there is no scope in microphysics for differences in "fitness" along the lines that molecules exemplify" (2005). Thus, if they introduce a new gap between physics and chemistry in order to reduce biological systems to chemical ones, they provide an explanation that is hardly solving the problem. They are just regressing from the first level at which the problem was posed, to another one.

\section{2- Physicalism and diachronic emergence}

"Not only is reification of worldviews a highly theoretical move of doubtful internal coherence, it stems from a radical misconception of the human condition" (Van Fraassen, 1999).

Rosenberg and Kaplan's vision of the PNS is based on the physical determination thesis that they emphasize ("physical facts fix all the facts"). This thesis is founded on two principles more explicitly developed by $\operatorname{Kim}$ (1993, 1998, and 1999): the causal closure and the completeness of the physical world. Rosenberg and Kaplan claim "that this thesis enjoys near universal acceptance" (2005) in philosophy of biology. Yet, we will argue that this thesis must be challenged in order to understand the very difference between real emergent biological phenomena and a very misleading application of the concept of supervenience in the field of evolutionary biology.

A- First, the physical determination thesis ("the physical facts fix all the facts") is a metaphysical claim, a "Weltanschauung". In other words, this is not a simple scientific

\footnotetext{
${ }^{5}$ The origin of this idea is in Feibleman (1954), see also Stengers (1997).
} 
assertion concerning a set of events that a scientist isolates, defined by kinds that facts instantiate and by laws that explain them. This proposition implies that the domain in which physical facts are ("all natural facts") is completely intelligible, even if we cannot describe them completely. This we can call the completeness of the physical world (CPW): "the idea that real connections exist and the idea that the world is intelligible and controllable are arguably equivalent ideas" (Kim, 1993: 53). We argue that this principle is based on a hidden logical fallacy.

From the CPW perspective, Kim jumps from the level of a finite system to the level of the world. How is it possible to make this jump? Obviously this cannot be done on science alone! And the answer is historically well known: one needs a Demon. Demons are everywhere in physics: Laplace, Maxwell, Boltzmann implicitly or explicitly used them. A physicist has a human eye, which symbolizes our epistemic limitation and the status of our scientific theories as instruments. Concerning the entire world, a physicist-as a physicist- can say nothing. Consequently, in order to become a physicalist -to reduce the real world itself to a set of physical events- the physicist needs a God's-Eye View (Putnam, 1990) ${ }^{6}$. He must employ an objective and impersonal entity, like the Laplacian demon. Yet, how can we accept the help of a supernatural entity on the one hand, if on the other we want to reduce the real world to a set of physical events?

One could counter that we can use the "physical determination thesis" without introducing a Laplacian Demon. We will answer, that it is impossible to justify "that physical facts fix all the facts" without to jump, "as if we were outside our own skins", from the level of the "facts" to the level of the world ("all the facts"). Furthermore, no connexion can be introduced between the idea of "unobservable entities", like atoms or electrons, and the idea of "the intelligibility of the world". We can try to give a limited representation of the electron with the help of experimental tools. This cannot be the case with respect to the "intelligibility of the world". Thus, we reject the CPW. Nobody is able to give a complete description of the world.

B- Rosenberg and Kaplan's thesis also implies that we can identify causal relations between natural facts that are physical ones. Physical facts fix the facts, meaning that biological or psychological facts cannot have any causal action in Nature. This means that the physical domain is not only intelligible, but also "causally closed". As conceptualised by Kim: "thus no causal chain involving a physical event will ever cross the boundary of the physical into the non-physical: if $\mathrm{x}$ is a physical event and $\mathrm{y}$ is a cause or effect of $\mathrm{x}$, then $\mathrm{y}$, too, must be a physical event" (1993: 147). In short, every physical event would have a physical cause: let's call it the Causal Closure Principle (CCP).

The physical determination thesis also means that the causal power of higher-level properties is reducible to the causal power of the base-level properties. Kim calls this the Causal Inheritance Principle (CIP, Kim 1993, 1999; Perovic, 2007). Accordingly, even if one accepts either synchronic or diachronic emergence, one must reject the principle of "the causal efficiency of the emergents" (Kim, 1999). Emergent properties could not have causal powers of their own. If causes would work simultaneously at the basal and at the higher levels, they would produce an overdetermination of the effect. If the instantiation of a property $M$ of an

\footnotetext{
6 "The same notion of a God'-Eye View"... the same epistemic ideal of achieving a view from an "Archimedean point" - a point from which we can survey observers as if they were not ourselves, survey them as if we were, so to speak, outside of our own skins - is involved" (Putnam, 1990: 17).

${ }^{7}$ (Putnam, 1990: 17).
} 
object $\mathrm{X}$ would cause the instantiation of another property $\mathrm{M}^{*}$, and if $\mathrm{M}$ would depend on a physical property $\mathrm{P}$ by which $\mathrm{M}$ is realized, or implemented, it would mean that, either $\mathrm{M}^{*}$ depends on another physical property $\mathrm{P}^{*}$, having no ontological efficiency, or $\mathrm{M}^{*}$ would modify a microproperty $\mathrm{P}$ in $\mathrm{P}^{*}$, which would violate the $\mathrm{CCP}$.

In this view, "emergence" could be accepted as an epistemic property involving unpredictability from lower levels as long as it is connected with mereological supervenience, like it seems to be the case for Rosenberg and Kaplan. But emergence could not be an ontological property, involving qualitative novelty (Bunge, 2003). By mereological supervenience, one means that "...systems with an identical total microstructural property have all over properties in common. Equivalently, all properties of a physical system supervene on, or are determined by, its microstructural property" (Kim, 1999). This view is compatible with the idea that there is a "complete microstructural description" (Kim, 1999) of the system, and that the upper-level conditions are not "resulting" from the basal-conditions. Macrostructural properties could be said to be unpredictable and in a certain way unexplainable. The relation between $\mathrm{M}$ and $\mathrm{P}$ is not univocal. $\mathrm{M}$ is depending on $\mathrm{P}$, or on $\mathrm{P}^{*}$, or on $\mathrm{P}^{* *}$. Yet with the same physical systems, one would always have the same supervenient macroproperties. Thus, physical facts would seem to fix the facts.

Bunge has discussed all the difficulties related to the use of this concept of supervenience (2003: 14). We will instead focus on the fact that the CCP and the CIP are founded on the $\mathrm{CPW}$. Given the incomplete description of the physical world, it would be impossible to assert that all physical events have a physical cause. This clears the way for a new conception of diachronic emergence compatible with downward causation.

C- In our view, a scientific theory in classical physics (not in non-linear dynamics or in thermodynamics of open systems far from equilibrium) is nothing but "an interpretation" of a finite system (even the universe is one), characterised by a certain class of theoretical models concerning the spatial coexistence and the temporal existence of physical events (Duhem, 1906, Van Fraassen, 1989, Longo 2006). We would add that even in quantum mechanics, a class of theoretical models is specified by its invariants or internal constraints, which are always finite and measurable. That's why the structure of such systems is not depending on time (Van Fraassen, 1989).

The determinism principle establishes that "the same causes produce the same effects": at T1 of a specific system (like the solar system) one can isolate initial conditions, laws and invariants, and based on them predict a final state at T2. This is not true of biological systems. Biological systems are not only finite, because they are not defined only by internal conditions or constraints, but they are open systems. A living organism has a metabolism, biochemical cycles, and it is sensitive to natural selection. External constraints are always operating on them. This has been called a "contextually non separable system" (Perovic, 2007). By non separability, Perovic assumes that a contextual open property $\mathrm{N}$ "is not instantiated in each individual constituent but rather in the context of the system's (individual) constituents" (Perovic, 2007).

Yet "non separability" is not only a property of the higher level that must be distinguished from those specifying the basal-level. We assume that the internal constraints defining a system are always disturbed by external ones, so that in order to understand what is going on in the system, we must jump simultaneously to multiple levels on which this system is integrated (Stengers, 1997). For instance, the cell, as a whole is integrated in a more complex 
system, the tissue, the organism, in which it will not act, as if it were placed in an in vitro culture. Let us take for example a muscle cell in the heart. The components of this system are proteins that channel the ions, (calcium, potassium), and they carry currents that changes the cell voltage. In turn the cell voltage changes the ion channels (Noble, 2006: 57). The components alter the behaviour of the heart and the heart alters the behaviour of the components, yet both components and the heart are integrated in a higher multicellular structure, the organism. This means that the working of such systems is never defined by initial internal constraints.

D- When dealing with open systems, new systemic properties emerge at T2 and they can modify the initial properties. Thus what was described at T1 was not the essence of the system. In other words, when one states that the biological facts at T1 cause physical facts at $\mathrm{T} 2$, and that they compete with the explanation of these facts as purely physical ones, we are making a mere idealisation. At $\mathrm{T} 2$, the system is not the same as the one at $\mathrm{T} 1$, because it has new properties that were not there at $\mathrm{T} 1$. Therefore, a system's description of natural events is not a complete description of what this system does. By diachronic emergence we mean the fact that in specific natural or formal systems the initial relations and properties of elements cannot teach us how they would be applied as the system evolves. Thus, the historical way by which a system of natural events operates is not a consequence of its description. It acts and it produces novelty (novel qualities and novel structures) in the real world, which leads to the conclusion that emergence has an ontological meaning (Bunge, 2003). It is not a simple epistemic property.

Consequently, we reject Rosenberg and Kaplan's argument against antireductionism because it introduces a conceptual confusion between emergence and supervenience. They note that, if physical facts at T1 fix the physical facts at T2, even if physical facts are biological facts at T1, it adds (ontologically) nothing to physical facts. They argue that, if this would not be true, contradictions would be inevitable. Our simple answer is: physical facts in a physical system (A) at T1 fix the physical facts at T2 in our representation of the system at T1. Nevertheless, our representation can never be a complete description of the system. This description can change by the mere fact that the system is working between T1 and T2. At T2, the system is historically changed, without any contradiction, since the real biological emergent properties were not present in our description, at $\mathrm{T} 1$. They are a result of the system's activity.

Dobhzansky's assertion that "nothing in biology makes sense except in the light of evolution" (1973) substantiates the view that diachronic emergence is a central property of biological systems. It suggests that predictions can only be achieved by means of reproductions of specific classes of phenomena. Diachronic emergence explains that irreversibility is a fundamental property of all evolutionary processes in biology. This irreversibility does not preclude that evolution is the result of computations represented by Boolean Networks. Admittedly, such computations are dynamic process by which new systemic properties emerge.

This brings about how computational sciences could contribute to the understanding of the concept of emergence. Let us recall an old and interesting example, i.e., Von Neumann's well-known model of reproductive automata (1961) that deals with the concept of complication. 


\section{3- Self-reproduction and self-replication}

In order to model reproduction we must understand how complication "becomes selfsupporting and even increasing" (Von Neumann, 1961). Degenerative complication is defined by the fact that an automaton A that has the ability to produce $\mathrm{B}$ needs a complete description of B plus "various arrangements in A that see to it that this description is interpreted and that the constructive operations that it calls are carried out." Then "a degenerative tendency must be expected" (Von Neumann, 1961). For instance, in the well known system of three automata (A, B, C), A constructs an automaton which is described by an instruction (i). B makes a copy of the instruction (i) that is furnished to it, and C combines A and B operations. This is illustrated below:

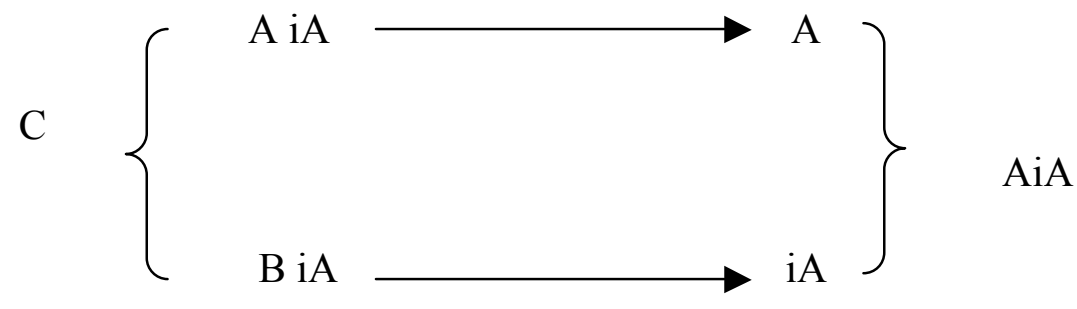

Let us take the aggregate $\mathrm{D}$ denoted by $\mathrm{A}+\mathrm{B}+\mathrm{C}$. The instruction to reproduce $\mathrm{D}$ and to make a copy of iD must be inserted in A and in B, respectively, so that:

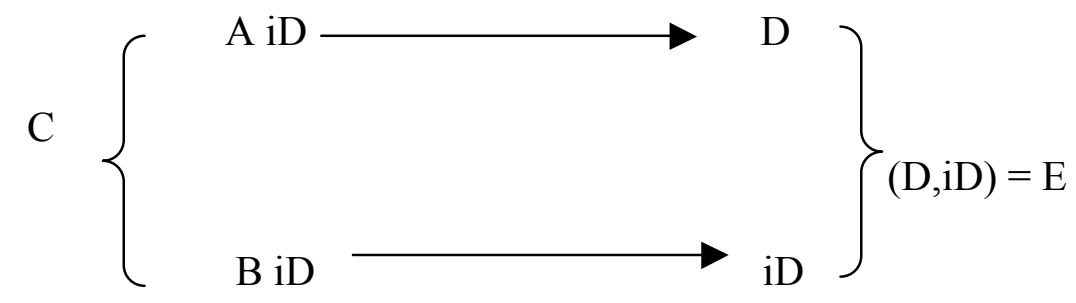

Von Neumann concluded that E is "clearly self-reproductive" without any vicious circle and "according to the rules of logic" and the complication of such a construction becomes "selfsupporting" and may even increase. The D system acquires the new systemic function of selfreproduction by the fact that $\mathrm{iD}$ is inserted in the aggregate (A, B, C), thus becoming $\mathrm{E}$. This transformation results from the fact that the description of $\mathrm{D}$ is different in this system from what is constructed with this description. $\mathrm{E}$ is a result of this construction and not an element of this description. E emerges in the sense of diachronic emergence. We can reproduce the same construction in a deterministic way and without mystery.

Let us now return to the problem of self-replication in experimental biology that is at the centre of Rosenberg and Kaplan's paper. They deal with the problem of knowing how selfreplication can be a result of catalytic activities of several molecules and not a fundamental property of a certain class of molecules. This means that auto-replication can be constructed in a reproductive way. Consider the example emphasized by Kauffman (2000) and experimented by Sievers and von Kiedrovski (1994) of a single stranded DNA hexamer that is replicated by a collective catalytic system. The hexamer A does not contain the genetic 
information to produce a copy of itself. Instead, the two triplets of A have the power to catalyse the ligation of $\mathrm{B}$ while the two triplets of $\mathrm{B}$ catalyse the ligation of A. Thus, reproduction is the result of this collective catalytic operation:

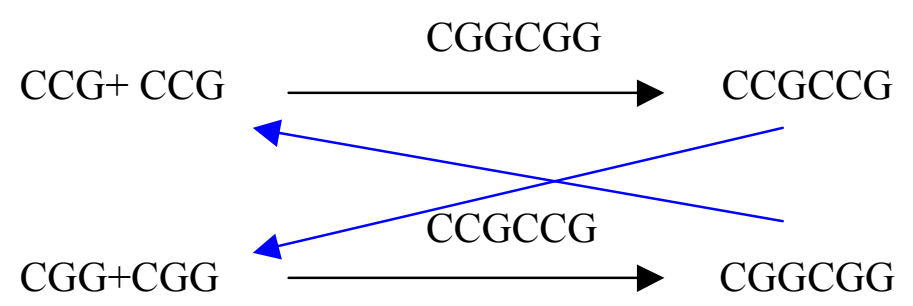

In the classical molecular model, a fragment of DNA codes for the synthesis of proteins by which DNA is replicated, thus falling in the well-known vicious circle whereby genes are translated into proteins with the help of proteins. In this novel way of thinking the circle becomes virtuous. In it, we have properties (stability or instability of molecules, auto and cross catalysis, etc.) and objects (DNA or RNA as ribozymes). Systemic properties, like selfreplication, result from what is constructed by the initial properties and objects. Therefore, the products cannot be elements of the initial description of the process. The first step is accomplished in order to understand how prebiotic genes can be inserted in a biological system.

\section{4- The entanglement enigma}

Assuming that systemic properties result from what is constructed from these properties and objects is valid, it implies that in the scientific methodology, one must take into account the action of downward causation. Let's come back to the origin of the concept in Kim's analysis. In our previous example (see Section 2c), the cell voltage is a systemic property. It depends on base properties by which it is realized (electric charged ions). Yet, Kim rejects the "causal efficacy" of systemic properties. According to Kim, emergent properties have no causal power of their own. Either the systemic property $\mathrm{E}$ is identified with the disjunction of its realizers (Q1 vs Q2 vs Q3...), or it is a "second order functional expression" by which we cannot generate new properties, "but only new ways of indifferently picking out, or grouping, first order properties" (Kim, 1999). However, in our example the cell voltage is a systemic property that changes the protein channel (which would be the definition of downward causation). This contradicts Kim because it leads to the idea that a macroproperty $\mathrm{M}$ of a system can add to the set of properties $\mathrm{Pi}$ of its elements a new property $\mathrm{Pj}$, and this violates the CCP and the CPW.

According to our view, to the contrary, downward causation is obviously possible since the natural world is not closed or completely described by any Demon. As we can say without any contradiction that ions are changing the cell voltage (upward); we can add that the change in cell voltage (systemic property) modifies the protein channels (downward), since both effects are not simultaneous, or were not simultaneous at the beginning. Kim neglects the fact that time is acting, and that this action of time is not an epistemic property. It is, instead, an ontological one. Let's consider Kim's celadon vase that is dropped from the window of his 
office (1999). Even, if we conclude that the vase, by virtue of its mass, has a set of causal powers that none of its micro-constituents have, the mass of this object is a resultant property. However, the electric voltage emerging from the activity of the ions is not a distributive property and it acts downward on the protein channel. Why is it so different from the celadon example? Our assumption is that time is acting on the system of elements considered as a whole modifying its internal structure, and thus the relation and the properties of its elements. This brings us to Noble's concept of "middle out", which is nothing but a heuristic tool for exploring downward causation in complex systems (Noble, 2003). A priori, one cannot distinguish, what is on the top and what is down, what is the system and what are its elements. In order to identify the elements, we need to understand first how the system has been modified by its history. They are no "elements per se", in the sense that they do not exist in isolation. They are "the elements" in a system modified by its history. That's why, on each level of explanation, we find strange entanglements in biology: genes are coding for the synthesis of proteins by proteins; the basal membrane is producing the architecture of tissues, yet the stroma and the epithelial cells are producing the basal membrane, etc (see section below).

We will argue that it is not downward causation what leads to these forms of reciprocal causation and to the entanglement enigma. We propose, on the contrary, that this enigma can be solved by the use of the concept of downward causation. Let us consider some biological examples in order to understand this point.

\section{a- Tissue interactions}

Since the introduction of the cell theory, most biologists readily accept that the cell is the unit of life. However, in multicellular organisms, single cells do not have an existence independent of the whole organism. These organisms and their cells are ontogenetically linked. Rather, a zygote - a cell resulting from the union of a female and a male gamete-divides, producing more cells, which are organized in a three-dimensional pattern. Both association patterns and cell types change as tissues and organs are formed. Hence, we consider multicellular organisms as complex systems in which the relations among parts are contextual and interdependent. From the beginning of ontogeny, each cell undergoes "differentiation" ${ }^{8}$ " under the influence of neighboring cells. This reciprocity makes it difficult to establish detailed cause and effect relationships. In addition, molecules are being sent from one cell to its surroundings. Such a molecule residing outside cells is no more a part of a cell. These extracellular molecules act on the immediate neighbors, or are carried into distant places by the bloodstream, where they modify the activity of yet other cells, which in turn produce molecules that react with other cells, and so on. These facts reveal that these hierarchical levels are entangled, at times precluding experimentally isolated cells from revealing their role in situ in the originating organism (Soto \& Sonnenschein, 2004).

Considering that cells constitute the "low level" parts of the higher level "tissue" or organism may not be always adequate. A tissue results from a long series of interactions during which cells move around in relation to one another and change in the process. By the time the tissue is formed, the "parts" that we identify in them are no longer the parts that interacted in their formation. The cellular components now present did not pre-exist the tissue itself-they are

\footnotetext{
${ }^{8}$ Differentiation : the sum of the developmental processes whereby from a single cell, the zygote the organism attains the diverse adult structures (cells, tissues and organs) and functions.
} 
interacting in a particular way that is reciprocal. When we artificially separate the components of the tissue, for instance the cells forming epithelium ${ }^{9}$ and its subjacent stroma ${ }^{10}$, cells cease to perform the functions they executed when together in their proper three-dimensional arrangement. However, when recombined, oftentimes they form a tissue similar to the one from which they originated. Parenthetically, this is an oversimplification because neither the parenchyma nor the stroma exists in isolation from one another. For example, recombining stroma and parenchyma ${ }^{11}$ (usually epithelium) from different organs has provided evidence about the inductive role of the stroma over the epithelium, as well as some indications that the epithelium possesses some degree of cellular identity that is not influenced by the stroma (Sakakura, 1979). This is illustrated by recombining fetal mammary gland stroma with salivary gland epithelium which results in a tissue that resembles the mammary gland regarding ductal structure. The recombination of salivary gland stroma with mammary epithelium results in a tissue that resembles a salivary gland. However, the epithelial cells in each case preserve their specific cellular response, i.e., mammary epithelial cells respond to the hormone prolactin by secreting milk proteins regardless of the provenance of the stroma.

As researchers tend to think that the important part of a tissue is its parenchyma, descriptions of changes occurring in the stroma are rare. However, the basement membrane that is interposed between the epithelium and the stroma is made "in collaboration" by the stroma and epithelial cells. Remarkably, some of those basement membrane proteins ${ }^{12}$ are produced by both cell types, while others are selectively produced. Nonetheless, the kinetics of production of each protein is different when the epithelium and the stroma are cultured in isolation, indicating that one cell type influences the output of the other cell type; that is, they influence each other reciprocally (Smola et al, 1998). The secreted proteins interact producing a highly organized structure. The epithelial cells form hemidesmosomes ${ }^{13}$, attaching themselves to the basement membrane. Notably, when epithelial cells are placed in a plain plastic culture dish, they arrange themselves, in a way that does not reflect the pattern of the epithelium of origin. If instead, they are placed in a similar dish coated with basement membrane proteins they associate and recover the original three-dimensional architecture of the epithelium of origin. They attach to each other forming either sheets or ducts. Moreover, the individual cell shapes also change, as does the intracellular placement of their organelles ${ }^{14}$. How can causation be studied here? Is the tissue causing the formation of a basement membrane? And then, is the basement membrane "causing" the normal architecture of the epithelium- and thus the tissue? This suggests downward causation.

\footnotetext{
${ }^{9}$ Epithelium: an avascular tissue that covers a free surface or lines-up a tube or cavity in an animal body and serves especially to enclose and protect the other parts of the body, produces secretions and excretions, and functions in assimilation (modified from: http:/www.nlm.nih.gov/medlineplus/mplusdictionary.html).

${ }^{10}$ Stroma: the supporting framework of an animal organ consisting of connective tissue (http://www.nlm.nih.gov/medlineplus/mplusdictionary.html).

${ }^{11}$ Parenchyma: the distinctive tissue of an organ or an abnormal growth as distinguished from its supportive framework (modified from: http://www.nlm.nih.gov/medlineplus/mplusdictionary.html).

${ }^{12}$ Basement membrane: a thin extracellular supporting layer that separates a layer of epithelial cells from the underlying lamina propria and is composed of the basal lamina and reticular lamina; Lamina propria: a highly vascular layer of connective tissue under the basement membrane lining a layer of epithelium (http://www.nlm.nih.gov/medlineplus/mplusdictionary.html).

${ }^{13}$ Hemidesmosomes: a specialization of the plasma membrane of an epithelial cell that serves to connect the basal surface of the cell to the basement membrane (modified from: http://www.nlm.nih.gov/medlineplus/mplusdictionary.html).

${ }^{14}$ Organelles: literally, organelle means "little organ"; they are specialized cellular parts (as a mitochondrion or ribosome) that perform the metabolic and synthetic functions of a cell.
} 
Tooth development offers another example of reciprocal causation. The mandibular epithelium "causes" the underlying mesenchyme ${ }^{15}$ to condense (i.e. it appears more cell-dense at the light microscope). If the mandibular epithelium is now isolated and recombined with mesenchyme from other organs, it induces the generation of tooth structures. Soon thereafter this "inductive" potential of the mandibular epithelium is lost. If the condensed mesenchyme from underneath the mandibular epithelium is now recombined with epithelia from other parts of the body, the recombinant generates tooth structures (Gilbert, 1997). Moreover, many gene products involved in early stages of tooth development, mammary gland development and in hair follicle development are similar. Hence, in order to understand how form is generated it may be more productive to concentrate on the higher-level phenomena (cell movement, cell proliferation, cell death) than exclusively in patterns of gene expression.

Thus, in the context of multicellular organisms, single cells are ontogenically linked. Epithelial cells and stromal cells jointly produce the basement membrane, while at the same time this membrane is producing the architecture of the tissue (stroma and epithelium). In this context, it is impossible to isolate gene or cell activity from tissue interactions. In order to understand the problem, one may start at the cell level, or at the level of tissue interactions; however, one will be forced to eventually move up and down levels of organization since multiple levels are participating in the phenomenon being explored. A complete explanation is out of reach because levels are entangled. In this context a useful heuristic tool would be to overcome the bottom-up and the top-down explanation by adopting the middle out approach proposed by Noble (2006). Since our ontological approach deals with open systems, all levels of organisation can be the starting point for a simulation. They can be many middles now, since the centre is everywhere and the circumference nowhere. Instead of explaining the higher level by the lower one, we can look for the lower level "by the filter of the higher level" (2006: 72).

This reciprocal causality is also central to the understanding of cancer. The cancer phenotype is not fixed from within the cancer cells (Sonnenschein and Soto, 1999). Cells from the inner cell mass of blastocysts (early embryos) grow as carcinomas when transplanted under the kidney capsule of a host. When these cells are transplanted back into normal blastocysts, they end up as normal cells in practically all the tissues of the mice that developed from those blastocysts (Mintz \& Ilmensee 1975). Similar normalization phenomena have been observed when liver carcinoma cells are transplanted into a normal liver (Coleman et al, 1997), when mammary carcinoma cells are transplanted into normal mammary gland stroma (Maffini et al, 2005) or when melanoma cells are placed into zebrafish embryos (Hendrix et al, 2007). However, the ability of the host organ to normalize cancer cells is influenced by developmental factors. For example, the ability of the liver to "normalize" liver cancer cells is more robust in young animals than in older ones, while the ability of the mammary stroma to normalize mammary carcinoma cells is enhanced by the age of the recipient and it is further enhanced if the animals have undergone pregnancy and nursed their offspring (Maffini et al, 2005).

The ontological stance of researchers is a major determinant of the models proposed to study a phenomenon. For decades, reductionists have interpreted the phenomenon of chemicallyinduced mammary cancer as due to a direct interaction between the carcinogen and the DNA of the "founder" epithelial cell of the resulting carcinoma. This stance overlooked two facts, namely, that the carcinogen was injected into a live animal, where all cells are exposed, and

\footnotetext{
${ }^{15}$ Mesenchyme: loosely organized mesodermal embryonic tissue that give rise to connective tissues. (modified from: http://www.nlm.nih.gov/medlineplus/mplusdictionary.html).
} 
that the carcinogen interacts with multiple macromolecules, in addition to DNA. By overlooking these facts, they exclusively searched for mutations in the DNA of the epithelial cells that became neoplastic. Only recently experiments were conducted to address whether the target of the carcinogen resided in the epithelium or in the stroma of the mammary gland. Using a theory-neutral experimental strategy, Maffini et al observed that the recombination of stroma exposed to a carcinogen with normal epithelial cells resulted in neoplasms (Maffini et $a i, 2004)$. The reverse combination did not. This observation suggests that the stroma, rather than individual cells in the epithelium, is the target of the carcinogen. These results also point to the contextuality of the neoplastic phenotype.

But where is this reciprocal causality coming from? The context of multicellular organisms is a product of history (evolution and ontogeny). We refuse all a priori views that predicate the possibility to see all different contexts, or all possible worlds, "instead of studying cases of emergence in the real world" (Bunge, 2003: 14). We are arguing then, that this contextdependence is an effect of diachronic emergence, and not the contrary.

Let us now look at a well-identified system of chemical objects that by interacting add new properties to this system. We take into account the system's history. Therefore new properties are acting and have the power to modify the system without any contradiction, or any infinite regression, since we don't need to assume that they could be described at the beginning. The new definition of the system must be done at another time, and it acts downward and not arbitrarily on the initial elements of the system. Let's relate a representative example from ontogeny in order to illustrate this point.

\section{b- An example in morphogenesis}

During embryogenesis the generation of form (morphogenesis) is mediated by cellular processes such as the direction and number of cell divisions, changes in cell shape, cell movement, cell growth, cell death and changes in the composition of the cell membrane and extracellular matrix (Gilbert, 1997). The reductionist view proposes that each of these cellular processes is generated by the differential expression of developmental genes. We consider instead, that in addition to this upward causation, cellular and tissue events occurring before the expression of a particular set of genes takes place may act downwardly modifying the expression of these genes at a later time (diachronic emergence). During gastrulation ${ }^{16}$ of the Drosophila embryo ${ }^{17}$, the mesoderm ${ }^{18}$ invaginates, i.e., it moves from the surface to the interior of the embryo, and the germ band ${ }^{19}$ extends (T1). This movement generates mechanical force that results in compression of the stomodeal cells (the anterior part of the alimentary canal; future mouth). As a result of this compression, the stomodeal cells express the gene twist (T2). This is a crucial event in the formation of the anterior gut. Mutant embryos that fail to undergo germ band extension also fail to express twist in the stomodeal cells. When these embryos are subjected to gentle compression, twist is expressed in the

\footnotetext{
${ }^{16}$ Gastrulation: after fertilization a series of rapid mitotic divisions (cleavage) result in the blastula. Next, the process of gastrulation takes place, whereby cells change positions with respect to each other; as the result of this process the germ layers (ectoderm, mesoderm and endoderm) are formed.

${ }^{17}$ Drosophila: a genus of small dipteran flies that include several (as D. melanogaster) that are extensively used in genetic research (modified from: http://www.nlm.nih.gov/medlineplus/mplusdictionary.html).

${ }^{18}$ Mesoderm: the middle of the three primary germ layers of an embryo that is the source especially of bone, muscle, connective tissue, and dermis; broadly speaking, tissue derived from this germ layer (modified from http://www.nlm.nih.gov/medlineplus/mplusdictionary.html).

${ }^{19}$ Germ band: a collection of cells along the ventral midline that includes all the cells that will form the trunk of the embryo (http://www.ncbi.nlm.nih.gov/books/bv.fcgi?indexed=google\&rid=dbio.section.1959).
} 
stomodeal cells and development continues. Furthermore, ablation of dorsal cells in normal embryos relieves compression during germ band extension; as a result, twist expression in the stomodeal cells fails to occur (Farge, 2003). This causal chain, from molecular events resulting in germ band extension to physical stress inducing the next molecular event (twist induction in stomodeal cells) represents an emergent event. In other words, a moving group of cells produces compression on another group of cells that respond to this force by expressing a gene product, twist, promoting in this way a downward causal chain. Reductionists may argue that this is not a truly downward cause, because an operator could also apply compression and generate the same outcome; however, the fact remains that during the process of organogenesis, the only option the embryo has to instantiate compression is by creating a three-dimensional structure that generates this force and hence, this outcome. From our perspective, it is clear that mechanical forces, in an abiotic system produce mechanical effects that are predictable. In a biological system, they may acquire novel properties, such as that of inducing gene expression, which cannot be predicted from our knowledge of the physical world. This novel property appears in a "tissue-world" whereby a mass of moving cells is needed to generate this force.

\section{Conclusion}

If we admit that a physical property $\mathrm{Pj}$ can be at the same time physical and biological as a result of the phylogenetic and ontogenetic history of organisms, all potential vicious circles are avoided. We have illustrated the problem with examples taken from organismal biology, specifically, embryonic development and carcinogenesis. When acting in a biological system such as an embryo during morphogenesis, a mechanical force acquires the new property of inducing the expression of a particular gene $(\mathrm{Pj})$. A simple chemical molecule, such as a carcinogen, has the new property to disrupt the architecture of a tissue and to initiate neoplastic development. In the context of new systemic properties, basic properties are changing. However, this new type of constraint is not fixed at the beginning. This is the meaning of downward causation (see section 4).

After a certain period of time $t$, a new macroproperty $M$ is emerging in a system of elements characterized by a set of physical properties Pi. Time is acting, not on a part of the system, but on the system conceived "as a whole". This action is real and has an ontological meaning. Thus the system, having $\mathrm{M}$ at $\mathrm{t}$, causes the new property $\mathrm{P}$ (this is our definition of downward causation). Note that Kim is curiously not in disagreement with this conception of "reflexive downward causation" based on the use of diachronic emergence (1999). Yet, it is more compelling when one rejects the $\mathrm{CCP}$, the $\mathrm{CPW}$ and the physical determination thesis. Thus, we deal with ontological emergence, in the sense that the action of time explains how the new property $\mathrm{M}$ is emerging. It is not only a question of prediction. It is not due to our limited cognitive capacities.

Downward causation makes philosophical sense only when it correlates with diachronic emergence. It requires that the system in which it appears be time-dependent. That is, the system is a process, not a thing. Downward causation leads to a vicious circle only in the context of synchronic emergence (Kim, 1999). This is so because if systemic properties depend on basic properties; the former cannot complete or modify the basic properties simultaneously without contradiction. 
This explains why, in order to understand what is going on in the system after a period of time $\mathrm{t}$, we must jump simultaneously to multiple levels on which this system is integrated (see section 2C). Genes are coding for the synthesis of proteins by the proteins. Proteins carry current that change the cell voltage. In turn the cell voltage changes the ion channels. Such a conclusion legitimates the heuristic tool proposed by Noble and by which both reductionist and holist approaches are rejected in the fields of developmental biology and phyisiology. "Middle out" is neither a bottom-up reconstruction, nor a top-down synthesis (see also Bunge 2003: 46-47). This new design allows us to understand how "the orchestra of life" works, and "how large it is" (Noble, 2006).

Our analysis also has evolutionary relevance. Diachronic changes can be simulated and natural selection can be used in this simulation (Kauffman, 2000). Yet this simulation will not explain how natural selection as a biological or a chemical property is emerging itself in the world, as Rosenberg and Kaplan propose. Moreover, Kaplan and Rosenberg's assertion by which natural selection is understood as a natural law is not obvious.

There is a difference between explanation and computation. Kauffman's automata have a fitness value in NK models, where $\mathrm{N}=$ automata and $\mathrm{K}=$ interactions. NK networks have little to do with classical models of maximisation in population genetics. What these systems of automata are doing is not explained by the description of their elements. New constraints are emerging by the process of computation that simulates evolution. Thus, we all have to deal again with the old French aphorism: "le temps est ce qui se fait, et même ce qui fait que tout se fait. ${ }^{20}$,

\section{Acknowledgements:}

We thank Professor Ulises Moulines (University of Munich) and Doctor Slobodan Perovic (Carleton University) for their critical reading and helpful comments concerning an earlier draft of this manuscript.

\section{References:}

Atlan H (1986) Emergence of Classification Procedures in Automata Networks as a Model of Self Organization. Journal of Biological Theory, 120: 371-80.

Bedeau M (1997) Weak emergence, Philosophical perspectives of mind causation and world : 375-99.

Black M (1976) Models and Metaphors - Studies in Language and Philosophy, Ithaca and London, Cornell Un. Pr., $6^{\text {th }}$ printing.

Bunge M (2003) Emergence and Convergence, Toronto University Press.

Cairns-Smith G (1982) Genetic takeover and the mineral origin of life. Cambridge University Press.

Coleman W, Wennerberg AE, Smith GJ, Grisham JW (1997), Regulation of the differentiation of diploid and aneuploid rat liver epithelial (stem-like) cells by the liver microenvironment. American Journal of Pathology 142: 1373-1382.

Dennett D (1996) Darwin's Dangerous Idea. Touchstone, NewYork.

\footnotetext{
20 'Time is what makes itself and even what makes everything [else] make itself.'
} 
Dobhzansky Th (1973) Nothing in biology makes sense except in the light of evolution, American Biology Teacher, 35: 125-129.

Duhem P (1906) La Théorie physique, son Objet, sa Structure. Chevalier et Rivière, Paris. Farge E (2003) Mechanical induction of twist in the Drosophila foregut/stomodeal primordium. Current Biology 13:1365-1377 .

Feibleman JK (1954) Theory of integrative levels. The British Journal of Philosophy of Science, 5, 17: 59-66.

Fisher R (1930) The Genetical Theory of Natural Selection, Clarendon Press, Oxford. Gatenby RA, Vincent TL., (2003) An evolutionary model of carcinogenesis, Cancer Res. 63:6212-20.

Gilbert S (1997) Developmental Biology. Sinauer Associates, Inc., Sunderland, 5 edn. Hendrix MJ, Seftor EA, Seftor RE, Kasemeier-Kulesa J, Kulesa PM, Postovit LM, (2007) Reprogramming metastatic tumour cells with embryonic microenvironments, Nat Rev Cancer. (4):246-55.

Kauffman S (1974) The large scale structure and dynamics of gene control circuits. Journal of Biological Theory, 44, 167-190.

Kauffman S (1993) The Origin of Order. Oxford University Press, Oxford.

Kauffman S (2000) Investigations, Oxford University Press, Oxford.

Kauffman S (2002) Simulation of a Chemical Autonomous Agent. Zeitschrift für Physikalisches Chemie, 216: 1-9.

Kim J (1993) Supervenience and Mind. Cambridge University Press.

Kim J (1998) Philosophy of Mind. Westview Press.

Kim J (1999) Making sense of Emergence. Philosophical Studies 95: 3-36.

Kitcher P (1984) 1953 and All That: A Tale of Two Sciences. Philosophical Review 93: 335-373.

Laforge B, Guez D, Martinez M et Kupiec JJ (2005) Modeling embryogenesis and cancer: an approach based on an equilibrium between the autostabilization of stochastic gene expression and the interdependence of cells for proliferation, Progress in Biophysics and Molecular Biology, 89: 93-120.

Levin SA (2003) Complex adaptative systems : exploring the known and the unknown and the unknowable Bull. Am. Soc., 40 (1): 3-19.

Longo G, Bailly F, (2006) Mathématiques et Sciences de la Nature. La singularité physique du vivant, Hermann, Paris.

Maffini MV, Calabro JM, Soto AM, Sonnenschein C (2005) Stromal regulation of neoplastic development: Age-dependent normalization of neoplastic mammary cells by mammary stroma. American Journal of Pathology 67: 1405-1410

Maffini MV, Soto AM, Calabro JM, Ucci AA, Sonnenschein C (2004) The stroma as a crucial target in rat mammary gland carcinogenesis. Journal of Cell Science 117:1495-1502. Mayr E (1959) Where are we? , Cold Spring Harbor Symposia on Quantitative Biology, 24, 58-64.

Mayr E (1961) Cause and Effect in Biology, Nature, 134, 1501-1505.

Miquel PA (2000) Comment penser le désordre? Fayard, Paris.

Mintz B, Ilmensee K (1975) Normal genetically mosaic mice produced from malignant teratocarcinoma cells. Proceedings of the National Academy of Science of the United States of America 72:3585-3589.

Noble D (2006) The Music of Life, Oxford University Press, Oxford.

Perovic S (2007) The Limitation of Kim's Reductive Physicalism. Acta Biotheoretica 55:

243-67.

Putnam H (1990) Realism as a Human Face, Harvard University Press. 
Rosenberg A, Kaplan DM (2005) How to reconcile Physicalism and Antireductionism about Biology. Philosophy of Science, 72: 43-68.

Sakakura T, Sakagami Y, Nishizuka Y (1979) Persistence of responsiveness of adult mouse mammary gland to induction by embryonic mesenchyme. Dev. Biol. 72: 201-210

Sievers D, Von Kiedrovski G (1994) Self-replication of complementary nucleotide-based oligomers. Nature, 369: 221-24.

Smola H Stark H-J, Gabi Thiekötter G, Mirancea N, Krieg T and Fusenig NE (1998)

Dynamics of Basement Membrane Formation by Keratinocyte-Fibroblast Interactions. In: Organotypic Skin Culture, Experimental Cell research, 239: 399-410.

Sober E (1984) The Nature of Selection: Evolutionary Theory in Philosophical Focus.

Cambridge, MA: MIT Press.

Sonnenschein C, Soto A (1999) The Society of Cells. Bios Scientific Publishers, Oxford.

Soto A, Sonnenschein C (2004) Emergentism as a default: Cancer as a problem of tissue organization. J. Biosci. 30: 103-118.

Stengers I, (1997) Cosmopolitiques VI, La découverte, Paris.

Stephan A (2002) Emergentism, irreducibility, and downward causation. Grazer

Philosophische Studien, 65: 77-93.

Stephan A (1999) Varieties of Emergentism. Evolution and Cognition, Vol 5, n 1, 51-54.

Turing A (1936) On computable numbers with an application to the Entscheidungsproblem.

Proc. London Math. Soc. Ser, 2, 42: 230-65.

Van Fraassen B (1989) Laws and Symmetry, Oxford University Press.

Van Fraassen B (1999) The Manifest Image and the Scientific Image, in D. Aerts (ed.).

Einstein Meets Magritte: The White Book -- An Interdisciplinary Reflection, Kluwer,

Dordrecht : 29-52.

Van Fraassen B (2004) Science as representation: flouting the criteria, Philosophy of Science, 71: 794-804.

Von Neumann J (1961) The General and Logical Theory of Automata, in Taub A H (ed) John von Neumann: Collected Works. Volume V: Design of Computers, Theory of Automata and Numerical Analysis, Pergamon Press, Oxford, 9: 288-328.

Weisbuch G (1989) Dynamique des systèmes complexes: une introduction aux réseaux

d'automates. Collection Savoirs Actuels, InterEditions/Editions du CNRS, Paris.

Wright S (1932) The Roles of Mutation, Inbreeding, Crossbreeding, and Selection. In: D.F. Jones (ed.) Evolution. Proceedings of the Sixth International Congress of Genetics: 356-366. Wright S (1977) Evolution and the Genetics of Population, T3. Univ. Chicago Press, Chicago. Wright S ( 1980) Genic and Organismic Selection, Evolution, 34: 825-843. 
